\title{
Effects of relearning sessions on verbal mediating responses'
}

The effects of repeated sessions upon learning the acquisition pairs and upon mediated facilitation were examined in a modified "miniature"-mediation situation. In Experiment I, two sessions were separated by 48-hr. In Experiment II, three sessions were separated by either 5-min. or 48-hr. The numbers of presentations of the pairs of the two acquisition stages required to meet a criterion decreased over the sessions but mediated facilitation did not. The inter-session interval did not influence either acquisition of the pairs or mediated facilitation.

In the typical study of experimentally-acquired verbal mediating responses Ss learn the acquisition pairs and then are tested for mediation. If the session were repeated so that Ss relearned the acquisition pairs and were retested for mediational responding, the acquisition pairs would be expected to reach criterion strength more rapidly during the relearning session than during the initial session but there is no a priori reason why mediational responding should increase so long as the acquisition pairs were mastered before each test for mediation was administered.

The present experiments were designed to study interand intra-session relationships using a modified "miniature"'-mediation situation. Two sessions, $48 \mathrm{hr}$. apart, were used in Experiment $I$, and three sessions, separated by either $5 \mathrm{~min}$. or $48 \mathrm{hr}$, in Experiment II. In Experiment I a "mediation-retention" test consisting of the test-trials only preceded the second session.

\section{Method}

Six first-stage pairs from mediation sets, B-C, and the tests of retention of these pairs (cue-trials) were randomly interspersed among six first-stage pairs from control sets, $\mathrm{X}-\mathrm{C}$, and their cuetrials in a mixed design. The Ss were instructed to spell aloud all trigrams and to provide the missing response term on the cue-trials. If $\mathrm{S}$ was unable to give the missing response, the pair was shown again, then the cue-trial was presented and so on until $S$ responded correctly to the cue. When $\mathrm{S}$ met the criterion of mastery for a particular pair-three successive cue-trials without prompting by re-presentation of the pair-the second-stage pair for the set, B-A for both mediation and control sets, and its cue-trial were substituted for the learned pair. When $S$ met the same criterion for the second-stage pair, the test trial for that particular set was presented. On the test trials, the response term from the second-stage pair, A, was used as the stimulus for three response alternatives, including the response term from the first-stage pair, C (A-CDE). The S's task was to select the response that made the "best pair" with the stimulus $A$. Mediated facilitation was assumed if the $\mathrm{C}$ alternative was chosen more often following mediation than following control presentation.

All Ss in Experiment I returned $48 \mathrm{hr}$. later. They were tested for mediation by displaying the 12 test trials (a "mediation-retention" test) in order of occurrence of the test trials during the initial session. A relearning session followed which was procedurally identical to the initial session. Two intersession intervals were used in Experiment II, 5-min. and 48-hr. No mediation-retention test was administered in Experiment II.
The materials were constructed from CVC trigrams of $87-100 \%$ association value (Underwood \& Schulz, 1960). The mediation sets corresponded to the response-equivalence paradigm, B-C, B, B-A, B, A-CDE. Twelve control sets were prepared by substituting a new trigram, $X$, for the stimulus trigram of the first pair, $B$. The basic control paradigm was $\mathrm{X}-\mathrm{C}, \mathrm{X}, \mathrm{B}-\mathrm{A}, \mathrm{B}, \mathrm{A}-\mathrm{CDE}$. The $\mathrm{C}$ trigram appeared equally often in the first, second, and third position among the response alternatives on the test trials and the $\mathrm{D}$ and $\mathrm{E}$ alternatives were $C$ responses from other sets. For all sets, the cue-trial followed immediately the initial presentation of a pair and immediately preceded subsequent presentations of the pair. The materials were the same for both experiments but the spacing of repetitions of pairs from the same set was more controlled in Experiment $\mathrm{II}^{2}$. The only obvious result of this procedural change was to increase the difficulty of learning the first-stage pairs of Experiment II.

Six mediation sets and six control sets were assigned randomly to one "list" and the remaining sets automatically became the second "list". The Ss were assigned randomly to the lists subject to the restriction that each list be used equally often. Thirty-six introductory psychology students at Indiana University served in Experiment I, and 48 in Experiment II. The Ss of Experiment II were assigned randomly to the two inter-session intervals.

\section{Results}

The mean number of exposures required to reach the criterion was computed for the acquisition pairs for each session. The minimum number of exposures, three, was dictated by the criterion measure. As shown in Figs. 1 and 2, fewer exposures were required in the relearning session(s) than in the initial session for both experiments, Experiment I $(F=126.44, \mathrm{df}=1 / 34, \mathrm{p}<.01)$, Experiment II $(F=217.87, \mathrm{df}=2 / 92, \mathrm{p}<.01)$. This savings effect was found for both types of presentation and for both first and second-stage pairs although the stage of the pairs interacted with the session. For both experiments, the first-stage pairs required more exposures than the second-stage pairs during the initial session, Experiment I $(t=3.57, d f=136, p<.01)$, Experiment II $(t=27.21, d f=324, p<.01)$, but were of about the same difficulty as the second-stage pairs during relearning sessions.

The stage of the pairs interacted with the type of presentation: the first-stage pairs learned under media-
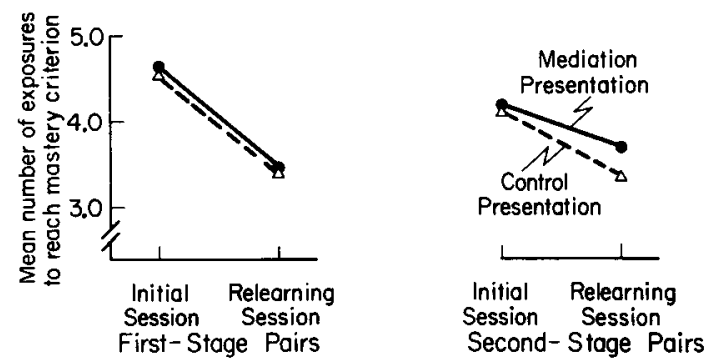

Fig. 1. Mean number of exposures to attain mastery criterion for first and second-stage pairs on the two sessions, Experiment $I$. 


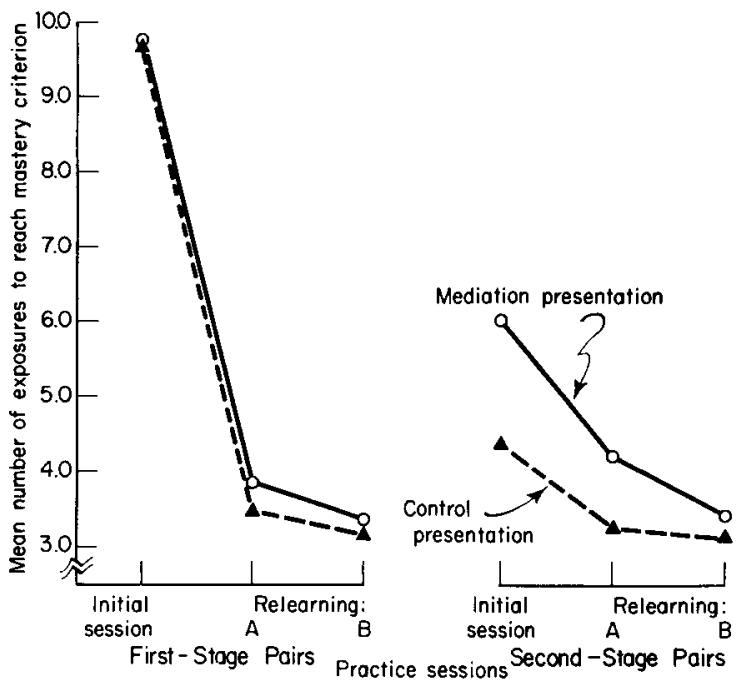

Fig. 2. Mean number of exposures to attain mastery criterion for first and second-stage pairs on the three sessions, Experiment II.

tion and control presentation required approximately the same mean number of exposures, but the second pairs under mediation presentation were more difficult to learn than the second-stage control pairs.

The mean number of selections of $\mathrm{C}$ on the test trials following mediation presentation exceeded the mean number of selections of $\mathrm{C}$ following control presentation for both experiments, Experiment $I$ ( $F=7.26$, $\mathrm{df}=1 / 34, \mathrm{p}<.05)$, Experiment II $(\mathrm{F}=11.00, \mathrm{df}=1 / 144$, $\mathrm{p}<.01$ ), providing evidence of mediated facilitation.

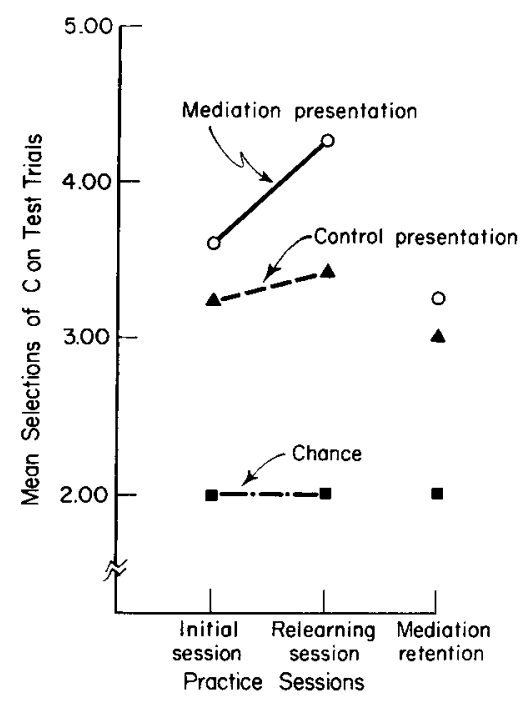

Fig. 3. Mean selections of $C$ on the test trials for the two sessions, Experiment I.

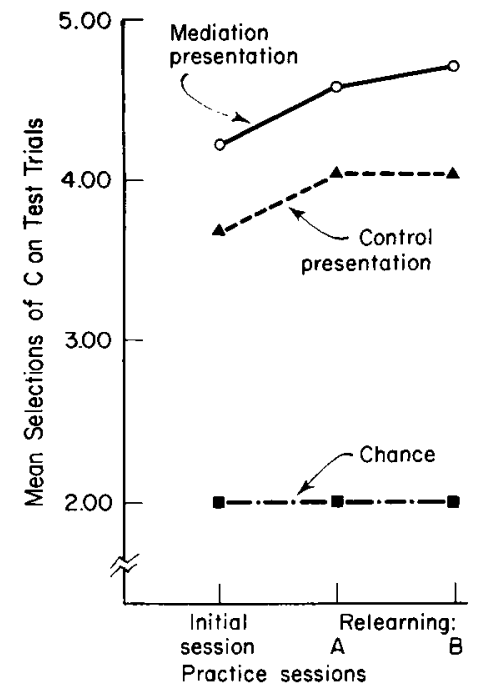

Fig. 4. Mean selections of $C$ on the test trials for the three sessions, Experiment II.

The mean selections of $\mathrm{C}$ on the test trials increased reliably from the initial to the relearning sessions, 3.43 and 3.84 , respectively, but, as shown in Fig. 3 and 4 , no interaction was found between the type of presentation and the sessions, Experiment $I(F=1.24, \mathrm{df}=2 / 68$, $\mathrm{p}<.05)$, Experiment II $(\mathrm{F}<1)$.

The mean selections of $\mathrm{C}$ on the mediation-retention test of Experiment I did not differ reliably.

The relationship between the number of exposures required by the two acquisition pairs of a set and the selection of $\mathrm{C}$ on the test trials was examined in two ways for both experiments. None of the analyses indicated a significant relationship.

\section{Discussion}

The number of exposures of the acquisition pairs required to meet the criterion declined markedly from session to session but mediated facilitation appeared to be relatively independent of the additional practice provided by the relearning sessions. Furthermore, mediated facilitation seemed to be unrelated to the ease of mastering the acquisition pairs (at least when the pairs were learned to a criterion) and to the variations in the inter-session intervals.

\section{Reference}

Underwood, B. J., \& Schulz, R. W. Meaningfulness and verbal learning. Chicago: Lippincott, 1960.

\section{Notes}

1. This research was supported by Public Health Service Research Grant MH 7944.

2. Details may be obtained from the authors. 